\title{
Focus on the patient is the central pillar of the digital transformation in health!
}

In 1903, the famous inventor Thomas Edison declared, in one of his many predictions about the future, that "the doctor of the future will give no medicine, but will interest his patient in the care of the human frame, in diet and in the cause and prevention of disease". He saw a long way!

This challenge to health is no surprise to some of us, yet still leaves us perplexed - we must persistently pursue the strategy of disease prevention rather than simply reacting to the treatment of those illnesses which occur and can be avoided. The perfect storm of factors that together make our current health services inaccessible and unsustainable is well known and well understood, given the number of studies published on the subject. Economic, epidemiological and demographic changes mean that the 'more of the same' formula is no longer able to deal with the many serious problems faced by health services in the 21st century ${ }^{1}$. Rising health care costs, consumer expectations, new technologies and increasing globalization have placed huge pressure on the health sector, forcing it to align more closely with the economic constraints that every country suffers.

Established long-term primary, secondary and tertiary care systems are increasingly unable to respond to the challenges arising from aging populations, as well as the shift in the burden of diseases to chronic conditions, such as diabetes and musculoskeletal and cardiovascular diseases.

The need for greater quality and efficiency in health care, not only in hospitals but also in the home, is becoming increasingly important for patients, especially those with chronic conditions, and for societies all around the world. The availability of new technologies, such as smartphones, wearable technology and cloud based electronic medical records, facilitates the development of more flexible and safer technological solutions for patients ${ }^{2,3}$

A recurring question is: will universalized digital medical record systems be able to transform our current health services? Put simply, YES. There are potential benefits for health management, with savings in treatment costs and a reduction in unnecessary spending. What we seek through this digital revolution in health is the virtuous sustainability of the system ${ }^{4}$

The report prepared for the NHS system in the UK, entitled the Topol Review - Preparing the Healthcare Workforce to Deliver the Digital Future ${ }^{5}$, published in February 2019, includes three principles to support the deployment of these digital technologies in healthcare. These are:

1. Patients should be adequately informed about health technologies, with particular focus on vulnerable groups to ensure fair access; 
2. Health professionals need knowledge and guidance to assess and adopt new technologies;

3. New technologies should be adopted to ensure that health teams have more time to care for and directly interact with patients. Technology should be a facilitator, never a barrier, in patient care.

The digital transformation in health seems to be a ready-made apparatus to allow the data generated by different technological innovations - as exemplified by the arrival of artificial intelligence in medicine - to offer possibilities to improve the safety of clinical trials, the surveillance of new and old diseases, the engagement of patients in the treatment prescribed and, consequently, lead to improvements in results, allowing a better quality of life for patients, from newborns to the growing generation of the elderly (a group that expects to live longer and with quality). The benefits are potentially huge, with obvious social and economic impacts.

Therefore, we must consider: are we preparing for this digital revolution in health? Will we be the agents of this transformation, or those who passively suffer it?

\section{Guilherme Machado Rabello}

Instituto do Coração-InCor

São Paulo, SP, Brasil.

\section{REFERENCES}

1. Marsch, L. A., Hegel, M. T., \& Greene, M. A. (2019). Leveraging digital technology to intervene on personality processes to promote healthy aging. Personality Disorders: Theory, Research, and Treatment, 10(1), 33-45. http://dx.doi.org/10.1037/ per0000275

2. Coughlin, S., Roberts, D., O’Neill, K. and Brooks, P. (2018), Looking to tomorrow's healthcare today: a participatory health perspective. Intern Med J, 48: 92-96. doi:10.1111/imj.13661

3. Kuo AMH (2011). Opportunities and Challenges of Cloud Computing to Improve Health Care Services. J Med Internet Res 2011;13(3):e67. doi:10.2196/jmir.1867

4. Richard Hillestad, James Bigelow, Anthony Bower, Federico Girosi, Robin Meili, Richard Scoville, and Roger Taylor (2005). Can Electronic Medical Record Systems Transform Health Care? Potential Health Benefits, Savings, And Costs. Health Affairs 2005 24:5, 1103-1117

5. Health Education England. The Topol Review, Preparing the healthcare workforce to deliver the digital future: an independent report on behalf of the Secretary of State for Health and Social Care February 2019 [Internet]. [sem local]: HEE; 2019 [acesso em 02 mar. 2019]. Disponível em: https://topol.hee.nhs.uk/wp-content/uploads/HEETopol-Review-2019.pdf 